\title{
Alzheimer Research Series on the Default Network
}

Pat McCaffrey, Tom Fagan and Esther Landhuis

Alzheimer Research Forum

\section{CORTICAL CONNECTIONS GET CLOSER LOOK IN HEALTH, DISEASE}

The idea of the default mode network, an interconnected set of brain regions that are active when the brain is resting and that power down during focused mental tasks, was first proposed in 2001, but it quickly became a hot topic in cognitive neuroscience and for Alzheimer's disease (AD) researchers. The seesaw activation and deactivation of the default network and task-related brain regions appears critical for peak performance on memory tasks and deactivation and network connectivity are disrupted early in $\mathrm{AD}$, perhaps as a result of amyloid deposition [1].

$\mathrm{AD}$ is not the only disease where network activity suffers. A new study in PNAS reveals that function of the default network, as measured by fMRI, is also altered in people with schizophrenia, and their healthy first-degree relatives [2]. The changes are somewhat different from those seen in $\mathrm{AD}$ - network connectivity is strengthened, for one, and overall network activity is increased both at rest and during task. The study, from Susan Whitfield-Gabrieli at MIT, suggests that changes in default mode activity and alterations in the normal balance between activation and deactivation contribute to the symptoms of schizophrenia, and could be part of the genetic risk for the disease.

To look at the default network in people with schizophrenia, the researchers performed fMRI scans on subjects while they were idle, and then when they engaged in a simple memory test. That allowed assessment of the basal network activity, and the extent of deactivation that occurred during a task that required concentration. The study compared 13 volunteers with early phase schizophrenia, 13 unaffected first-degree relatives and 13 healthy controls. When subjects performed the test, suppression of the default mode network was most effective in controls, and decreased in both patients and relatives. Overall, the deactivation in the default network and activation of task-related areas were strongly correlated in control subjects, but the seesaw effect was much weaker in patients and relatives. The result was a consistent hyperactivity of the default network. In addition, the patients and relatives showed stronger connectively between the medial prefrontal cortex and the precuneous and the rest of the default network, whether measured at rest or during a task.

The strength of connectivity and defect in deactivation correlated with poorer performance in the working memory task and stronger schizophrenia symptoms, suggesting that the default mode network could play an important role in the cognitive and clinical symptoms of schizophrenia. In addition, the observation that unaffected relatives show similar changes suggests that aberrant network activity stems from genetic risk and is causal, rather than just a consequence of the disease, Whitfield-Gabrieli told ARF. "In the future, it may be possible to use these fMRI measures as a way of diagnosing disease, or to figure out how patients are responding to treatment," she said.

In terms of the symptoms of schizophrenia, it is interesting that the default mode network is activated during internal, self-referential thought. Network overactivity could lead to problems integrating the internal and external worlds, the authors speculate. Besides AD and schizophrenia, other diseases where default mode network activity is known to be altered include autism, 
epilepsy, depression, and attention deficit/hyperactivity disorder [3].

\section{Connect the neurons}

Networks like the default mode involve widely distributed areas of the brain, but no matter how far-flung their components may be, the basic unit of any network comes down to neuron-to-neuron communication at individual synapses. A detailed map of the brain would greatly help researchers understand how default and other networks related to neurologic disease, and such a map requires an understanding of how individual neurons are wired to each other. However, it has been nearly impossible to tease out the exact contacts that make up even simple networks from the spaghetti-like tangle of axons, dendrites, and cell bodies that make up brain tissue. Now, three different groups have used sophisticated imaging techniques and single cell recording to get over that hurdle, and each has a paper in Nature revealing the connections that make up cortical neuron networks in mice.

First, Solange Brown and Shaul Hestrin of Stanford University looked at the local connections among three classes of cortical neurons that project over long distances to the contralateral cortex, the contralateral striatum, or the superior colliculus [4]. After retrograde labeling of projections in live mice to distinguish the different kinds of neurons, the researchers simultaneously recorded activity in sets of four cells in cortical slices. By stimulating each cell in turn and watching the others' responses, Brown and Hestrin showed they could identify local cortical connections with high success. The tendency of cells to make local connections was not random, or simply based on proximity, but depended on the identities of both the presynaptic and postsynaptic cells For example, a neuron that projected to the contralateral cortex (a corticocortical neuron) was four times more likely to make a synapse with a local corticotectal neuron than with another corticocortical neuron. The results suggest a way to unravel the local circuit architecture in the cortex.

In a second paper [5], researchers from Matthew Larkum's laboratory at the University of Bern in Switzerland used fiber optic imaging to measure dendritic calcium changes and uncover a cortical inhibitory microcircuit in living rats. In the circuit, they found inhibitory interneurons govern the graded calcium response in L5 pyramidal neuron dendrites after sensory stimuli. The results help explain how neurons can re- spond to sensory input over a large dynamic range by utilizing cortical micronetworks.

Finally, Karel Svoboda and colleagues [6] of the Howard Hughes Medical Institute in Ashburn, Virginia, traced out an excitatory circuit using photoactivation of neurons expressing channelrhodopsin [7] to map out points of contact. Taking cortical slices with channelrhodopsin expressed in axons, first author Leopoldo Petreanu and colleagues used a laser beam to stimulate local neurotransmitter release while simultaneously recording from barrel cortex pyramidal neurons. If the axons made a synapse on the recorded cell, a postsynaptic excitatory current would be triggered. By directing channelrhodopsin expression to different layers of the cortex whose axons overlap with the pyramidal cell dendrites of the barrel cortex, the investigators were able to map input from the thalamus, cortical layers $4,2 / 3$, and part of the motor cortex to the dendrites of L3 and L5 pyramidal neurons. They found many of the known connections, as well as some new ones. In addition, the mapping revealed a high degree of spatial specificity, with different inputs mapping onto distal versus apical locations on the dendrites. By allowing the high-resolution look at neuronal circuits, the technique should help achieve a micro-scale, comprehensive picture of the hardware of the brain. - Pat McCaffrey.

\section{MEASURING GROSS BRAIN CHANGES IN AD, FTD}

PNAS describes some new gross anatomical and electrophysiological changes that accompany AD and frontotemporal dementia (FTD). For one, researchers led by Klaus Linkenkaer-Hansen at VU University, Amsterdam, the Netherlands, report that $\alpha$ and $\vartheta$ brain waves in mild to moderate AD patients exhibit subtle changes in timing [8]. In the authors' view, the changes support the idea that default networks are compromised in $\mathrm{AD}$, and they could also serve as a marker to monitor disease progression or treatment. For another, Maria Gorno-Tempini and colleagues in the U.S. and Italy used MRI scans to examine the relationship between ApoE status and brain atrophy in $\mathrm{AD}$ and behavioral variant FTD (bvFTD) [9]. While there is an established link between the $\varepsilon 4$ variant of ApoE and brain atrophy in $\mathrm{AD}$, the researchers now extend this link to bvFTD as well, supporting the idea that ApoE4 may be a risk factor for multiple neurodegenerative diseases. 
The Dutch brain wave study employed magnetoencephalography (MEG) rather than the more common electroencephalography (EEG). "Electric fields can give rise to a potential on the scalp that makes it difficult to determine where the signals come from," Linkenkaer-Hansen told ARF. With MEG, the source of the signal can be more accurately pinpointed, indeed closely enough to allow this technology to guide neurosurgical treatment of intractable epilepsy and brain tumors. MEG is also relatively unobtrusive and fast. It does not require a multitude of electrodes taped to the scalp; instead, the magnetic field is measured by a helmet-type device that the participant can slip on and off in seconds. A typical MEG recording takes three minutes compared to the 15 minutes required for an EEG. "From the time the patient walks into the room to the time they leave is about 10 minutes," said Linkenkaer-Hansen. Speed is clearly an advantage when dealing with AD patients, who can get agitated during testing conditions.

The researchers took MEG recordings of 19 people with mild to moderate $\mathrm{AD}$ and 16 age-matched controls, and assessed temporal changes in different brain waves. It is established that amplitudes of different brain waves, including $\alpha, \gamma$, and $\vartheta$ bands, are different in $\mathrm{AD}$ patients compared to controls [10]. What joint first authors Teresa Montez, Simon-Schlomo Poil, and colleagues investigated was not simply the amplitude in such bands, but how they are modulated temporally. "Normal EEG and MEG analysis throw out all temporal information in the signal," explained LinkenkaerHansen. By contrast, he showed that normal volunteers generate oscillatory activity that has refractal character in time. This essentially means the oscillation can somehow carry a memory of its own dynamic for several tens of seconds [11]. "I suspected that it is important for memory that you have this coordination of brain activity over time," he said.

In this study, the researchers show that this temporal dynamic breaks down in AD patients. For example, $\alpha$ band (in this case $6-13 \mathrm{~Hz}$ ) oscillations in the parietal region normally bias activity in the same region several tens of seconds later in normal people, but this bias is significantly reduced in AD. The authors suggest that the "temporal structure is at least as important as the magnitude of the oscillations as a marker of pathophysiology and, possibly, mnemonic operations." In contrast, the authors found that temporal bias was significantly and greatly strengthened among $\vartheta$ frequency $(4-5 \mathrm{~Hz})$ oscillations emanating from the medial prefrontal cortex in AD patients compared to controls.
"Our interpretation is that this might reflect some sort of compensatory mechanism," said Linkenkaer-Hansen. There is growing evidence for compensatory mechanisms in $\mathrm{AD}$, including hippocampal hyperactivation and increased frontal activity.

Finally, the authors suggest that these MEG recordings support the concept that default networks - a baseline neural activity that occurs while people do not focus on a given mental task - is gone awry in people with dementia. $\vartheta$ oscillations are part and parcel of this default network activity [12].

Previous EEG studies have found differences in brain wave amplitudes between people with dementia and controls [13], and even correlated those differences with hippocampal atrophy [14] and ApoE genotype [15]. Several magnetic resonance imaging studies, as well, have linked ApoE status with rates of brain atrophy in AD patients [16,17]; however, only one small study has examined the relationship between ApoE status and atrophy in FTD [18]. Now, researchers led by Maria Gorno-Tempini at the University of California at San Francisco report analysis of a larger FTD imaging study focusing on patients with behavioral variant FTD (bvFTD), also called Pick's disease. This is the most common form of FTD and is characterized by altered social behavior, emotions, and self-awareness. The study indicates that bvFTD patients who also carry the ApoE4 allele have greater brain atrophy in select regions of the brain than bvFTD patients who do not carry that particular isoform of the apolipoprotein. ApoE4 is already a known risk factor for this type of FTD [19].

First author Federica Agosta and colleagues used voxel-based morphometric (VBM) analysis to measure brain atrophy in 51 people with AD, 31 with bvFTD, and 51 controls. Consistent with previous imaging studies, they found greater atrophy in hippocampus and parietal cortex in ApoE4-positive AD patients. In the bvFTD group, Agosta and colleagues found that ApoE4-positive patients had greater atrophy in regions of the brain that typically degenerate in this disease, most notably both sides of the anterior cingulate cortex, and a broad region of only the right frontal cortex. Other small areas that appeared to atrophy more in ApoE4 carriers than non-carriers include the right caudate, superior temporal gyri, and left frontal gyri. The results suggest that ApoE4 somehow influences the underlying pathology of FTD. According to the authors, that might explain some curious observations. For example, one concerned a pathological dichotomy between two first-degree relatives who both had FTD - 
the brother who was homozygous for ApoE4 had more profound behavioral and cognitive problems than his sibling, who carried two copies of ApoE3.

Whether ApoE affects the progression of bvFTD is unknown. In AD, it is generally accepted that E4 brings on the disease earlier and speeds it up. The authors had not set out to address this question in this study, but while they saw no correlation between cognition and ApoE status, they do write that "Certainly, the atrophy patterns in our VBM study suggest that the FTD $\varepsilon 4$ carriers may be at higher risk for rapid clinical decline." This question requires a longitudinal study. Tom Fagan.

\section{CORTICAL HUBS FOUND CAPPED WITH AMYLOID}

In any social circle, there are always one or two key people who seem to be the center of the action. They have many friends, keep up with everyone's news, remember birthdays, and instigate events. Social psychologists and network theorists give these butterflies the designation hubs because they link otherwise disconnected people to each other and provide the glue that keeps the group together. As it turns out, the brain also has hubs - regions in the cortex that reach out and touch many otherwise separate processing units. The hubs function to coordinate and integrate the workings of different parts of the brain.

Using functional MRI (fMRI), Randy Buckner, with Reisa Sperling, Keith Johnson, and colleagues, all at Harvard University, have mapped in fine detail a small set of cortical hubs, regions that are the most highly and stably connected to other parts of the brain [20]. What's more, they show that in patients with $A D$, the pattern of amyloid accumulation tracks to these hub areas. The work, published in the Journal of Neuroscience, suggests that the intrinsic network architecture of the brain may be part of the reason why certain areas accumulate amyloid. One possibility is that the hubs represent regions of intense neuronal activity and/or metabolism that promote $\mathrm{A} \beta$ production, a conjecture that remains to be proven.

To find the hubs, Buckner and colleagues analyzed spontaneous fluctuations in the fMRI BOLD (bloodoxygen-level dependent) signal across the brains of healthy young adults. The measure of functional connectivity is defined by a voxel-to-voxel correlation in the BOLD signal in different brain regions over time. The researchers looked for regions that had the most

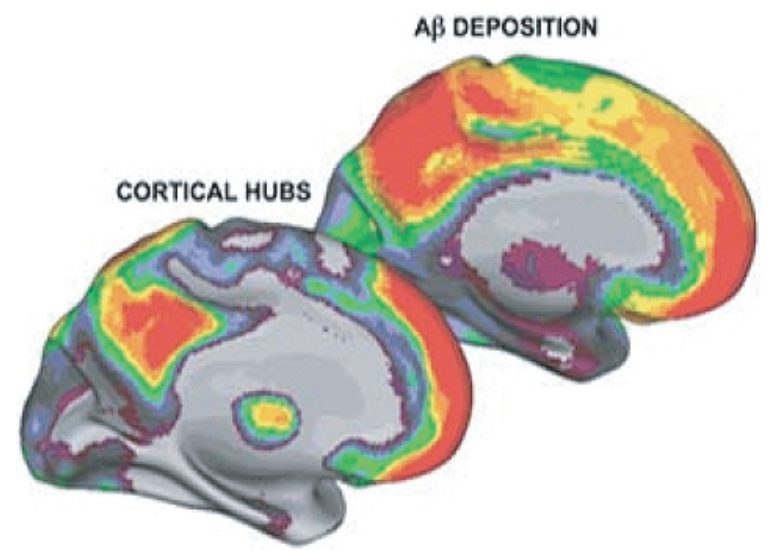

Fig. 1.

correlation with multiple other parts of the brain, and identified a set of hubs in the posterior cingulate, lateral temporal, lateral parietal, and medial/lateral prefrontal areas of the cortex. The hubs showed up whether the subjects were resting or were performing a challenging word test, suggesting that they are a stable part of the brain architecture. By pooling fMRI data on 127 subjects, the researchers generated a consensus reference map of the cortical hubs.

The second part of the study compared the distribution of hub activity to that of amyloid in people with $\mathrm{AD}$ as measured by PET imaging using Pittsburgh compound B (PIB). The results showed a visually apparent and computationally significant correlation between the two.

New study strengthens link between brain activity and amyloid

A human study combining functional connectivity imaging of the brain with amyloid imaging has added a new twist to the rapidly evolving science of the brain's default mode network. The study suggests, ominously perhaps, that something about the intrinsic architecture of our brain renders the most densely connected regions - the hubs of activity - vulnerable to persistently high $\mathrm{A} \beta$ production. The foreground image shows cortical hubs as measured by fMRI in young healthy adults. The background image shows amyloid deposition measured in AD using PIB-PET. The two show strong correspondence. Image credit: Jorge Sepulcre and Randy Buckner, Harvard University and MGH

Interestingly, the five core hubs lie in the default mode network, a set of interconnected brain regions that show suppressed activity when the brain is active- 
ly engaged with the external world, and have higher activity when people are daydreaming, or musing to themselves. Buckner's previous work showed that the default network was a preferential site for amyloid deposition, and other studies have established that disruption of this network even in the resting state is a marker for early AD. These results led to the speculation that the level of background activity in the default network, which hums along when the brain is not otherwise occupied, could account for the preferential accumulation of $\mathrm{A} \beta$ in those regions as a result of the activity-dependent processing of amyloid precursor protein.

The new work says something slightly different. The strong correlation between amyloid deposition and hub activity suggests that $\mathrm{A} \beta$ deposition does not depend on what the brain is doing in terms of cognitive work, but instead is a feature of the underlying architecture of the brain. The hub areas show the greatest fluctuations in BOLD signals at all times, and, Buckner said, "The assumption is that those signals are related to high levels of metabolism and high levels of net activity."

The relationship between BOLD oscillations, neuronal activity, and $\mathrm{A} \beta$ production remains to be demonstrated, but Dennis Selkoe of Harvard Medical School favors the same model. "I do believe this provides further indirect evidence for neuronal activity increasing $\mathrm{A} \beta$ generation via activity-dependent processing of $\mathrm{A} \beta \mathrm{PP}$, in accord with the nice work of John Cirrito and colleagues in recent years," Selkoe told ARF [21].

If hub activity relates directly to $\mathrm{A} \beta$ production, then this raises the possibility that individual variations in connectivity could be a risk factor for $\mathrm{AD}$, or that known risk factors might affect hub activity. This will be a focus for future work, as Buckner explains further in a Q\&A with ARF (below). - Pat McCaffrey.

\section{Q\&A WITH RANDY BUCKNER. QUESTIONS BY PAT MCCAFFREY}

Q: How did you conduct this study, and what does it show?

A: Our basic approach was to identify regions of the brain that show disproportionately high numbers of connections to other brain regions. We sought to identify the way stations of information processing in the brain. The brain has a number of specialized systems. Anatomists such as Marsel Mesulam and others have long postulated that there are special regions, called heteromodal areas or association cortex, that connect many of these specialized brain systems together, and this might be why we are able to think thoughts that rely on multiple systems, and how we are able to integrate visual and auditory information. In our paper, we specifically looked for the regions of the brain that are most connected - we call these hubs because they have numerous connections to diverse areas of the brain.

The way we did this is to look at the spontaneous or intrinsic activity in the brain, which has been a focus of many recent research papers on AD such as from Michael Greicius' laboratory at Stanford. In this case we used spontaneous activity as a tool to look at connections, because we think that these spontaneous activity events cascade among anatomically connected regions. So if you want to understand which regions are anatomically connected and which ones have the most connections in the human, characterizing intrinsic activity networks provides a reasonable approach. To do this, we took a large group of research volunteers and simply made a map of those cortical regions that showed the most connectivity across the rest of the brain. Using this approach we were able to map the brain's cortical hubs.

I should mention a caveat. An assumption of our work is that the spontaneous activity fluctuations that we can measure with fMRI are constrained by anatomic connectivity. However, we have pretty good reason to believe there is not a precise one-to-one relationship between functional connectivity and anatomic connectivity. fMRI seems to be sensitive to both direct or indirect anatomic connections. A paper published from Olaf Sporn's group at Indiana University confirms the suspicion that functional connectivity is more pervasive than direct anatomic connectivity [22,23].

Q: What does the connectivity map look like?

A: The first important finding was that there is a network of regions that are the most connected in the brain. We did not know in advance what to expect, although we had our suspicions based on classic work by anatomists who have characterized areas of association cortex. We discovered that the network of regions that was disproportionately connected was highly similar to the default network, those regions which are most active when people think to themselves, as compared to doing an active task.

Q: Why is that?

A: Why do we use these heteromodal hubs when we think to ourselves more than other systems such as sensory systems? That's an interesting question and we briefly write about it in a few paragraphs of the paper. What this study tells us is that the regions of the brain that we spontaneously use when we think to ourselves 
are also those that are convergence zones. We really do not have a good answer yet as to why the two converge on the same network. But even without a clear answer, I suspect it is not a coincidence.

Q: And the connection to AD...?

A: Using amyloid imaging based on PIB, we were further able to show that there is a strong correlation between the map of these hubs and where amyloid deposits in $\mathrm{AD}$.

This is related to our earlier work on the default network in some ways - showing a relationship between the topography of the network in young adults and the development of $\mathrm{AD}$ - but extends it in an important way. In the past we had been focusing on different cognitive states such as thinking to ourselves as the possible origin of why these regions that show heightened activity and metabolism may be conducive to AD, but this work now shows that it may be a more fundamental property of the network's architecture that leads to the heightened activity and heightened metabolism. The network contains regions that are highly interconnected across the brain and show the highest level of fluctuations on fMRI most of the time, during rest states and also during task states. That is, while the activity fluctuations in these regions are heightened during rest, they persist during active states as well. This property may lead to high levels of neuronal activity and high levels of metabolism. I would like to see cellular-level work in this area to help us understand better the relationship between these regions of high functional connectivity and tonic activity or heightened metabolism.

Q: Is the idea that the high neuronal activity leads to enhanced $\mathrm{A} \beta \mathrm{PP}$ processing and production of $\mathrm{A} \beta$ ?

A: Yes, that is what the extraordinary work from Dave Holtzman's laboratory at Washington University, and John Cirrito's work in particular, has taught us. Using a transgenic mouse model of AD, they demonstrated that transient changes in neuronal activity lead to increases in $\mathrm{A} \beta$ [21]. I suspect that these are all related observations, but we need further study to help establish what the relationships are.

Mark Mintun and Marc Raichle, also from Washington University, presented findings a few years ago at the Society for Neuroscience meeting that suggest glycolytic metabolism may play a special role in deposition of amyloid. It will be interesting to explore whether heightened activity in the functional connectivity hubs is what underlies regional differences in glycolysis. Pathways associated with glycolytic metabolism may reflect a related, or even alternative, route by which activity hubs might influence the progression of $\mathrm{AD}$ pathology.
Q: What are the ramifications of that for thinking about AD in terms of diagnosis or treatment?

A: The work of Michael Greicius at Stanford and Cindy Lustig at the University of Michigan has already shown that you can use disruption of this network as a marker for AD [24-26]. Our new work may help to understand why this network and this particular set of connectivity paths are related to amyloid deposition and subsequently to disruption of the network. This may help to answer the question of why these regions are particularly vulnerable and why their disruption may be a marker for AD.

Our findings also raise another important possibility that relates to risk for $\mathrm{AD}$. We are presently exploring whether there are differences between people in these functional connectivity patterns. It is possible that the measure developed in the paper, which is effectively a measure of the pervasiveness of functional connectivity in the brain, may differ across individuals. If it does, we would like to know if those differences relate to genetic factors that affect how the brain is wired during development. And, if there are genetic factors that relate to these patterns of connectivity, are they risk factors for $\mathrm{AD}$ ?

Q: Any insights about treatment?

A: Understanding pathways that link activity and metabolism to amyloid deposition, or other cascades that are involved in AD neurotoxicity, will hopefully identify targets that one can manipulate. Based on our findings, one can imagine that future preventative treatments might target early life events that tweak the relationship between brain activity and $\mathrm{A} \beta \mathrm{PP}$ production or brain activity and other cascades important to AD. The findings we report and those recently emerging from other laboratories push us to consider the possibility that normal lifelong cellular processes modulated by brain activity are the foothold for AD.

Current treatments have focused on the late-stage processing of $\mathrm{A} \beta \mathrm{PP}$ and the deposition of $\mathrm{A} \beta$. What we are seeing is initial evidence that there might be earlier stages of the process that could yield treatment candidates, although we have not yet identified what those candidates might be.

\section{APOE4 LINKED TO DEFAULT NETWORK DIFFERENCES IN YOUNG ADULTS}

Whether intensely focused or thinking of nothing in particular, people carrying the ApoE4 allele show differences in brain activity at a very young age, accord- 
ing to a study published in PNAS. Researchers led by Clare MacKay at the University of Oxford, U.K., report that ApoE4 carriers have distinct patterns of activity in the "default mode network," a group of brain areas that preferentially succumb to atrophy and amyloid deposition in AD [27]. Whether the changes seen in young ApoE4 carriers relate to $\mathrm{AD}$ is not clear, but the new data suggest that ApoE4 does something to the brain decades before the possible onset of memory loss or neuropathology in these at-risk individuals.

The default network - a set of brain regions that perk up when the mind is adrift and tone down during periods of mental focus - has become a hotbed for brain imaging research [28]. These cortical areas are among those most highly connected with other parts of the brain. In AD patients, they rack up the most amyloid [29] and show disturbed activity [30]. Young ApoE4 carriers have abnormally low rates of glucose metabolism in some default-mode regions [31]. In the current study, first author Nicola Filippino and colleagues complement the glucose utilization findings with fMRI data.

A previous investigation by Adam Fleisher, then at the University of California, San Diego, and colleagues, picked up ApoE4-linked differences in older people during task-induced fMRI [32]. The new analysis extends those findings to younger adults and includes resting-state fMRI measurements, which some think may better reflect the brain's intrinsic functional connectivity - how it is wired when not engaged in a specific task. "Inclusion of resting-state imaging is critical to understanding baseline pathophysiology and is quickly becoming utilized in the AD fMRI community," Fleisher wrote in an e-mail to ARF. He is now the associate director of brain imaging at Banner Alzheimer's Institute in Phoenix, Arizona.

For the new study, the researchers recruited 36 healthy people between the ages of 20 and 35. Half of the participants carried an ApoE4 allele but otherwise matched the non-carrier group in gender, age, education, and memory performance. Key differences between the two groups showed up in brain imaging data from blood oxygen level-dependent (BOLD) fMRI. In scans taken while participants were asked to recall familiar versus novel images, the ApoE4 group had higher hippocampal activation, consistent with previous studies of older ApoE4 carriers [33,34]. Even while resting in a dark room without being asked to focus on anything, the young ApoE4 carriers in the new study showed different brain activity: they had higher activation in various parts of the default network (retrosplenial, medial temporal, and medial-prefrontal cortex regions) compared with non-carriers. Curiously, these patterns ran counter to the changes documented in patients with $\mathrm{AD}$ [35] or with mild cognitive impairment (MCI) [36], who have dampened activity in default-mode areas.

That discrepancy suggests to Bill Rebeck "that ApoE genotype has an effect on the brain independent of any effects on AD pathological changes." Rebeck is a neuroscientist at Georgetown University Medical Center, Washington, DC, who was not involved with the new study. Considering the young age of the participants, it is hard to rationalize that the differences seen in ApoE4 carriers were due to effects on plaques and tangles, Rebeck noted in an e-mail to ARF. The new study supports a scenario in which ApoE4 may influence normal brain functions, "and then when AD pathological changes begin later in life, the two (E4 and AD) synergistically interact to drive the AD processes forward more dramatically," he wrote.

Coupled with a recent study showing that ApoE4 gene dose tracks with amyloid load revealed by positron emission tomography, the new investigation uncovers the potential for brain imaging techniques to serve as biomarkers of disease in treatment trials for AD," Fleisher noted. "Development of these techniques may play a key role in discovery of a cure for AD." - Esther Landhuis.

\section{NETWORK CONNECTIONS: MISSING LINKS IN NEURODEGENERATION?}

A story that grew rapidly from flicker to flame in $\mathrm{AD}$ circles over the past few years has been what role an important brain network might play in the early stages of disease. The default mode network, identified by the synchronized activity of its component parts on fMRI scans, is a target for amyloid deposition.

But what about other neurodegenerative diseases? Do they also spread through distributed networks? Could that explain the distinct patterns of pathology in different diseases? The answer appears to be yes, based on new data from William Seeley and colleagues at the University of California, San Francisco, and Michael Greicius at Stanford University. In a paper in Neuron [37], the researchers report how they used MRI to compare the patterns of atrophy and network activity in $\mathrm{AD}$ and four other diseases. In each case, the patterns delineated a different functional network. The results paint a picture of the selective vulnerability of different networks to different pathological insults, and sug- 
gest that neurodegeneration may propagate via network connections.

To compare different diseases, the researchers analyzed a total of 102 patients with one of five distinct dementia syndromes, including AD, three types of frontotemporal dementia (behavioral variant frontotemporal dementia, semantic dementia, and progressive nonfluent aphasia) and corticobasal syndrome. To better age-match healthy controls, and to focus on the beginnings of disease, the scientists chose young patients who had early-onset disease, and excluded patients with moderate or severe dementia.

Using first structural MRI, the researchers found that the patterns of atrophy for each condition were distinct, as previously described, and corresponded to the different cognitive deficits noted in each patient group. To probe for possible network connections, Seeley and colleagues chose the brain region that showed the greatest atrophy for each condition, and looked for synchronized activity with that seed region during a task-free fMRI scan in healthy controls. They found that each of the five disease-associated regions anchored a different functionally connected network. Moreover, in each case the network activity mapped closely to the distribution of atrophy observed in the analysis of diseased brains.

The functional linkage of brain regions was mirrored in structural measures, as well. Analyses of gray matter intensity across the whole brain in both normal and disease subjects revealed that the functionally linked regions also showed a high correlation of gray matter volume. As Seeley explained to ARF, "This suggests that regions that fire together, scale together. They grow and shrink together, although we don't know exactly how that works." Adding a structural correlation to the functional measures may be a useful approach to define networks in general, Seeley says. This is similar to previous work that used correlations of cortical thickness to map the default network [38].

The results support the "network degeneration hypothesis," which holds that disease starts in small neuron populations and progressively spreads to connected areas. "The point is that once a disease gets going at a given location in the brain, the whole network or regions that are interconnected with that region are at risk, and the disease is likely to propel itself down that set of pathways somehow or the other," Seeley explained. Because the study looks at different clinical syndromes that can be caused by a number of pathogenic proteins, this ability to propagate through networks is likely to be relevant to numerous disease proteins, including $\mathrm{A} \beta$, tau, $\alpha$-synuclein, and TDP43. How the proteins might spread their damage through networks is not clear, but there are several possibilities. Pathogenic proteins like $\mathrm{A} \beta$ are known to disrupt synaptic connections directly, and this could weaken networks. Some disease-causing proteins disrupt axonal transport, possibly leading to a severing of connections between regions. Some might even propagate misfolding by direct transfer from one neuron to another [39]. These potential scenarios are not mutually exclusive, the authors point out. And the extensive overlap between neurodegenerative diseases, for example, AD and dementia with Lewy bodies (DLB), both clinically and pathologically, is likely to complicate this new line of research as it digs deeper.

The network map for AD agrees with previous work showing involvement of the default-mode network, but the other diseases had their own distinct patterns. That suggests that the network signatures might eventually find use clinically to diagnose early stage or even presymptomatic disease, when there are still neurons to save. "The nice thing about network analyses is that they don't rely on atrophy," Seeley said. "We could potentially see the disease signature before brain regions start to shrink and wither." The measure might also serve as an objective biological endpoint to monitor disease progression and treatment. Researchers including Greicius and Randy Buckner are already working on developing these methods for AD, and the new data should spur studies of similar approaches for other diseases.

An outstanding question remains as to why different diseases, and presumably different pathogenic proteins, have a favorite network to attack. "Each disease, defined at the protein level, has a set of neurons somewhere in the brain that are most vulnerable to that protein's misfolding. That's the concept of selective vulnerability, and understanding what brings these problems to these cells is critical to understanding neurodegenerative disease," Seeley said.

To that end, Seeley says he is glad to see the concepts of network biology becoming more interwoven into the fabric of neurodegeneration research. "So much of the basic research on neurodegeneration focuses on single cells and molecules, but the tide is turning on that a little bit. We're seeing a new wave of network-oriented basic and translational neuroscience to try to understand what it is about network physiology, or gene expression, or development that makes these systems disease vulnerable. I'm excited to see that pick up." The importance of pushing forward with explorations of network structure and function in the human brain was echoed 
in an accompanying commentary by Marsel Mesulam of Northwestern University in Chicago, Illinois, who pioneered anatomical study of networks important for behavior and cognition [40]. - Pat McCaffrey.

\section{BOLD NEW LOOK - A $\beta$ LINKED TO DEFAULT NETWORK DYSFUNCTION}

By now, the curious detection of brain $\mathrm{A} \beta$ deposits in some healthy seniors has become commonplace among amyloid imaging practitioners. Whether this amyloid portends future dementia isn't so clear, but a report in the July 30 Neuron puts meat and bones on the hunch that it does [41]. "I think this is the first time we have strong evidence that amyloid is associated with brain dysfunction prior to symptoms," said first author Reisa Sperling, Massachusetts General Hospital, Boston, in an interview with ARF. A team led by her and husbandcolleague Keith Johnson, also at MGH, has found that brain $\mathrm{A} \beta$ pathology correlates with abnormal activity in memory networks of non-demented elders. The skewed network signals, picked up by fMRI, were "very similar to what's been observed in patients with mild cognitive impairment (MCI) and early stages of AD," Sperling said. "This, to me, is evidence that the process of $\mathrm{AD}$ does indeed begin many years before you get symptoms and that these individuals with amyloid really might be in the prodromal stages."

Using Pittsburgh compound B (PIB) and positron emission tomography (PET) to image fibrillar brain amyloid in vivo, scientists have found $\mathrm{A} \beta$ deposition in 10 to 40 percent of cognitively healthy seniors in various recent investigations, including the current one. Sperling and colleagues studied 18 young people (ages 18-30) and 35 older adults (ages 60-90). Thirteen of the older participants had subjective memory complaints and were thus classified with a Clinical Dementia Rating (CDR) of 0.5. However, none of these people demonstrated memory impairment on neuropsychological tests, nor did they meet MCI criteria. As such, the study population as a whole was considered "nondemented." Among the older participants, regardless of CDR designation, roughly 30 percent came up PIBpositive - that is, they had brain amyloid loads within a range typical for $\mathrm{AD}$ patients.

The big issue, as Sperling put it, is the "black box between amyloid and the cognitive symptoms you see in AD." Dating back decades to early autopsy studies in the field, amyloid plaques - despite being one of the best-characterized pathological hallmarks of $\mathrm{AD}$ - have correlated poorly with measures of brain function. There are people who have widespread amyloid deposits in their brains but show no signs of impairment. In an attempt to establish a functional link, Sperling's team looked to the default network, a set of interconnected brain areas that fire up when the mind is at rest and tone down during focused mental tasks.

This network has risen to fame in AD research with reports of aberrant default mode activity in people with early AD [42], in MCI patients challenged with visual and memory tasks [43], and in young adults carrying the $\mathrm{AD}$ risk allele ApoE4 [44]. What's more, two brain areas most prone to $\mathrm{A} \beta$ deposition - the medial prefrontal cortex and precuneus/posterior cingulate are both part of the default network [45]. And last year, Sperling and colleagues showed that the extent to which the brain areas of the default network tone down, or deactivate, during memory formation determines a person's ability to recall that learned information later [46]. In that study, the researchers measured blood-oxygen-level-dependent (BOLD) fMRI changes in young and old adults while they learned face-name pairs and while they recalled them a half hour later.

For the new work, the scientists used a modified version of that memory task and similar fMRI methods, but also included PIB-PET scans to determine if the people showing disrupted default network activity during the memory test were the ones who also had high levels of brain amyloid. In short, the answer was yes. Among the older participants, increased amyloid load associated with diminished deactivation in the precuneus/posterior cingulate. The correlation between amyloid burden and default mode deactivation held similarly in participants with and without subjective memory complaints. "It didn't make as much of a difference whether you were a CDR 0 or 0.5 as it did whether you had amyloid," Sperling said. "Amyloid was what was associated with the abnormality."

However, though amyloid load seemed to track with disrupted fMRI activity during the memory task, the researchers found no overall correlation between PIB retention and actual task performance. This "may not be surprising," the authors write, given that all of the older participants were still performing in the normal range, i.e., did not show impairment in standard neuropsychological measures, which include memory components. In an e-mail to ARF, Sperling explained that the facename task in the current paper was not as difficult as the one her laboratory had used previously [46]. Her group speculates that cognitive reserve may also explain why subjects performed well despite having large amounts 
of brain amyloid. Analyses are ongoing to tease out this possibility.

Something more intriguing, perhaps, was that the PIB-positive seniors showed a net increase in fMRI activity while engaged in the memory task (as opposed to PIB-negative seniors, who just showed decreased deactivation but still a net decrease in fMRI activity).

One speculation is that their revved-up brain activity helps compensate for amyloid-related network disruptions. However, it "might be a sign that the system is starting to break down," Sperling said, noting that this pattern of paradoxically increased fMRI activity is also seen in later stages of $\mathrm{AD}$ and in MCI $[47,48]$. "That makes it less likely to be compensatory and more likely a marker that something's about to fail," she said.

Bill Jagust, University of California, Berkeley, author of a recent review on amyloid imaging [49], appears on board with this line of thinking. In an accompanying review of the new study, he writes that it "adds a second phenotypic marker characteristic of $\mathrm{AD}$ to the first marker of $\mathrm{A} \beta$ deposition, supporting the view that those individuals with $\mathrm{A} \beta$ and functional alterations in the default mode network are in the early stages of AD despite normal cognition."

Others are more hesitant about this conclusion. "To my mind, what they very elegantly show is that amyloid plaques affect BOLD signal," said Scott Small of Columbia University, New York, in an interview with ARF. "I would probably be a little more cautious than they were in saying that $\mathrm{A} \beta$ affects underlying neural activity." The problem with any form of fMRI is that it is only an indirect measure of neural activity, he said, noting that recent work supports "an alternative interpretation - that the effect plaques have on the BOLD response is independent of underlying 'neural activity' but rather reflects changes in baseline flow or vascular reactivity." Sperling said that additional studies relating amyloid load to glucose metabolism and baseline tissue perfusion are underway and should help discern whether those properties are affecting BOLD signal in the current study. For now, though, "what I can say is that this finding is pretty specific to when people are performing the memory task well - when they are attempting to learn a face-name pair, and when they recall it successfully," she said. To her, this makes it less likely that the fMRI measurements are skewed by baseline differences.

Assuming the BOLD changes do reflect brain dysfunction, Sperling said the most exciting application of the new data is the possibility of using fMRI or other functional measures, along with amyloid imaging, as biomarkers in clinical trials of amyloid-modifying drugs. "This study suggests to me that we really can link amyloid and memory impairment, and that we have markers to detect this before the point of irreversible damage," she said, noting that continued longitudinal follow-up of the current participants is the only way to prove that brain $\mathrm{A} \beta$ in fact leads to memory impairment. In the meantime, the existing data already provide "evidence that we might be able to use markers like this in early drug trials," she said. "Then we have a window in which to ask whether we are affecting the disease, without having to wait three years to see who develops cognitive impairment."

\section{REFERENCES}

[1] Elsinghorst PW, Härtig W, Goldhammer S, Grosche J, Gütschow M (2009) A gorge-spanning, high-affinity cholinesterase inhibitor to explore beta-amyloid plaques. Org Biomol Chem 7, 3940-3946.

[2] Whitfield-Gabrieli S, Thermenos HW, Milanovic S, Tsuang MT, Faraone SV, McCarley RW, Shenton ME, Green AI, Nieto-Castanon A, LaViolette P, Wojcik J, Gabrieli JDE, Seidman LJ (2009) Hyperactivity and hyperconnectivity of the default network in schizophrenia and in first-degree relatives of persons with schizophrenia. Proc Natl Acad Sci U S A 106, 1279-1284. Erratum in: Proc Natl Acad Sci U S A (2009) 106, 4572.

[3] Broyd SJ, Demanuele C, Debener S, Helps SK, James CJ, Sonuga-Barke EJ (2009) Default-mode brain dysfunction in mental disorders: A systematic review. Neurosci Biobehav Rev 33, 279-296.

[4] Brown SP, Hestrin S (2009) Intracortical circuits of pyramidal neurons reflect their long-range axonal targets. Nature 457, 1133-1136.

[5] Murayama M, Pérez-Garci E, Nevian T, Bock T, Senn W, Larkum ME (2009) Dendritic encoding of sensory stimuli controlled by deep cortical interneurons. Nature 457, 1137 1141.

[6] Petreanu L, Mao T, Sternson SM, Svoboda K (2009) The subcellular organization of neocortical excitatory connections. Nature 457, 1142-1145.

[7] Wang H, Peca J, Matsuzaki M, Matsuzaki K, Noguchi J, Qiu L, Wang D, Zhang F, Boyden E, Deisseroth K, Kasai H, Hall WC, Feng G, Augustine GJ (2007) High-speed mapping of synaptic connectivity using photostimulation in Channelrhodopsin-2 transgenic mice. Proc Natl Acad Sci U S A 104, 8143-8148.

[8] Montez T, Poil S-S, Jones BF, Manshanden I, Verbunt JPA, van Dijk BW, Brussaard AB, van Ooyen A, Stam CJ, Scheltens P, Linkenkaer-Hansen K (2009) Altered temporal correlations in parietal $\alpha$ and prefrontal $\vartheta$ oscillations in early-stage Alzheimer disease. Proc Natl Acad Sci U S A 106, 1614-1619.

[9] Agosta F, Vossel KA, Miller BL, Migliaccio R, Bonasera SJ, Filippi M, Boxer AL, Karydas A, Possin KL, Gorno-Tempini ML (2009) Apolipoprotein E4: disease-specific effects on brain atrophy in Alzheimer's disease and frontotemporal dementia. Proc Natl Acad Sci U S A 106, 2018-2022.

[10] Jackson CE, Snyder PJ (2008) Electroencephalography and event-related potentials as biomarkers of mild cognitive im- 
pairment and mild Alzheimer's disease. Alzheimers Dement 4(1 Suppl 1), S137-S143.

[11] Linkenkaer-Hansen K, Nikouline VV, Palva JM, Ilmoniemi RJ (2001) Long-range temporal correlations and scaling behavior in human brain oscillations. J Neurosci 21, 1370-1377.

[12] Gusnard DA, Raichle ME, Raichle ME (2001) Searching for a baseline: functional imaging and the resting human brain. Nat Rev Neurosci 2, 685-694.

[13] Moretti DV, Fracassi C, Pievani M, Geroldi C, Binetti G, Zanetti O, Sosta K, Rossini PM, Frisoni GB (2009) Increase of theta/gamma ratio is associated with memory impairment. Clin Neurophysiol 120, 295-303.

[14] Babiloni C, Frisoni GB, Pievani M, Vecchio F, Lizio R, Buttiglione M, Geroldi C, Fracassi C, Eusebi F, Ferri R, Rossini PM (2009) Hippocampal volume and cortical sources of EEG alpha rhythms in mild cognitive impairment and Alzheimer disease. Neuroimage 44, 123-135.

[15] Kramer G, van der Flier WM, de Langen C, Blankenstein MA, Scheltens P, Stam CJ (2008) EEG functional connectivity and ApoE genotype in Alzheimer's disease and controls. Clin Neurophysiol 119, 2727-2732.

[16] van de Pol LA, van der Flier WM, Korf ES, Fox NC, Barkhof F, Scheltens P (2007) Baseline predictors of rates of hippocampal atrophy in mild cognitive impairment. Neurology 69, 14911497.

[17] Hämäläinen A, Grau-Olivares M, Tervo S, Niskanen E, Pennanen C, Huuskonen J, Kivipelto M, Hänninen T, Tapiola M, Vanhanen M, Hallikainen M, Helkala EL, Nissinen A, Vanninen RL, Soininen H (2008) Apolipoprotein E epsilon 4 allele is associated with increased atrophy in progressive mild cognitive impairment: a voxel-based morphometric study. $\mathrm{Neu}$ rodegener Dis 5, 186-189.

[18] Boccardi M, Sabattoli F, Testa C, Beltramello A, Soininen H, Frisoni GB (2004) APOE and modulation of Alzheimer's and frontotemporal dementia. Neurosci Lett 356, 167-170.

[19] Engelborghs S, Dermaut B, Mariën P, Symons A, Vloeberghs E, Maertens K, Somers N, Goeman J, Rademakers R, van den Broeck M, Pickut B, Cruts M, van Broeckhoven C, De Deyn PP (2006) Dose dependent effect of APOE epsilon4 on behavioral symptoms in frontal lobe dementia. Neurobiol Aging 27, 285-292.

[20] Buckner RL, Sepulcre J, Talukdar T, Krienen FM, Liu H, Hedden T, Andrews-Hanna JR, Sperling RA, Johnson KA (2009) Cortical hubs revealed by intrinsic functional connectivity: mapping, assessment of stability, and relation to Alzheimer's disease. J Neurosci 29, 1860-1873.

[21] Cirrito JR, Yamada KA, Finn MB, Sloviter RS, Bales KR, May PC, Schoepp DD, Paul SM, Mennerick S, Holtzman DM (2005) Synaptic activity regulates interstitial fluid amyloidbeta levels in vivo. Neuron 48, 913-922.

[22] Honey CJ, Sporns O, Cammoun L, Gigandet X, Thiran JP, Meuli R, Hagmann P (2009) Predicting human resting-state functional connectivity from structural connectivity. Proc Natl Acad Sci U S A 106, 2035-2040.

[23] Bullmore E, Sporns O (2009) Complex brain networks: graph theoretical analysis of structural and functional systems. Nat Rev Neurosci 10, 186-198.

[24] Greicius MD, Srivastava G, Reiss AL, Menon V (2004) Default-mode network activity distinguishes Alzheimer's disease from healthy aging: evidence from functional MRI. Proc Natl Acad Sci U S A 101, 4637-4642.

[25] Greicius M (2008) Resting-state functional connectivity in neuropsychiatric disorders. Curr Opin Neurol 21, 424-430.
[26] Lustig C, Snyder AZ, Bhakta M, O’Brien KC, McAvoy M, Raichle ME, Morris JC, Buckner RL (2003) Functional deactivations: change with age and dementia of the Alzheimer type. Proc Natl Acad Sci U S A 100, 14504-14509.

[27] Filippini N, MacIntosh BJ, Hough MG, Goodwin GM, Frisoni GB, Smith SM, Matthews PM, Beckmann CF, Mackay CE (2009) Distinct patterns of brain activity in young carriers of the APOE-e4 allele. Proc Natl Acad Sci U S A 106, 7209-7214.

[28] Buckner RL, Andrews-Hanna JR, Schacter DL (2008) The brain's default network: anatomy, function, and relevance to disease. Ann N Y Acad Sci 1124, 1-38.

[29] Buckner RL, Sepulcre J, Talukdar T, Krienen FM, Liu H, Hedden T, Andrews-Hanna JR, Sperling RA, Johnson KA (2009) Cortical hubs revealed by intrinsic functional connectivity: mapping, assessment of stability, and relation to Alzheimer's disease. J Neurosci 29, 1860-1873.

[30] Buckner RL, Snyder AZ, Shannon BJ, LaRossa G, Sachs R, Fotenos AF, Sheline YI, Klunk WE, Mathis CA, Morris JC, Mintun MA (2005) Molecular, structural, and functional characterization of Alzheimer's disease: evidence for a relationship between default activity, amyloid, and memory. $\mathrm{J} \mathrm{Neu}$ rosci $25,7709-7717$.

[31] Reiman EM, Chen K, Alexander GE, Caselli RJ, Bandy D, Osborne D, Saunders AM, Hardy J (2004) Functional brain abnormalities in young adults at genetic risk for late-onset Alzheimer's dementia. Proc Natl Acad Sci U S A 101, 284-289.

[32] Fleisher AS, Houston WS, Eyler LT, Frye S, Jenkins C, Thal LJ, Bondi MW (2005) Identification of Alzheimer disease risk by functional magnetic resonance imaging. Arch Neurol 62, 1881-1888.

[33] Bookheimer SY, Strojwas MH, Cohen MS, Saunders AM, Pericak-Vance MA, Mazziotta JC, Small GW (2000) Patterns of brain activation in people at risk for Alzheimer's disease. $N$ Engl J Med 343, 450-456.

[34] Bondi MW, Houston WS, Eyler LT, Brown GG (2005) fMRI evidence of compensatory mechanisms in older adults at genetic risk for Alzheimer disease. Neurology 64, 501-508.

[35] Greicius MD, Srivastava G, Reiss AL, Menon V (2004) Default-mode network activity distinguishes Alzheimer's disease from healthy aging: evidence from functional MRI. Proc Natl Acad Sci U S A 101, 4637-4642.

[36] Sorg C, Riedl V, Mühlau M, Calhoun VD, Eichele T, Läer L, Drzezga A, Förstl H, Kurz A, Zimmer C, Wohlschläger AM (2007) Selective changes of resting-state networks in individuals at risk for Alzheimer's disease. Proc Natl Acad Sci U S A 104, 18760-18765.

[37] Seeley WW, Crawford RK, Zhou J, Miller BL, Greicius MD (2009). Neurodegenerative Diseases Target Large-Scale Human Brain Networks. Neuron 62, 42-52.

[38] He Y, Chen Z, Evans A (2008) Structural insights into aberrant topological patterns of large-scale cortical networks in Alzheimer's disease. J Neurosci 28, 4756-4766.

[39] Frost B, Ollesch J, Wille H, Diamond MI (2009) Conformational diversity of wild-type tau fibrils specified by templated conformation change. J Biol Chem 284, 3546-3551.

[40] Mesulam M (2009) Defining neurocognitive networks in the BOLD new world of computed connectivity. Neuron 62, 1-3.

[41] Sperling RA, LaViolette PS, O'Keefe K, O'Brien J, Rentz DM, Pihlajamaki M, Marshall G, Hyman BT, Selkoe DJ, Hedden T, Buckner RL, Becker JA, Johnson KA (2009) Amyloid deposition is associated with impaired default network function in older persons without dementia. Neuron 63, 178-188.

[42] Greicius MD, Srivastava G, Reiss AL, Menon V (2004) Default-mode network activity distinguishes Alzheimer's dis- 
ease from healthy aging: evidence from functional MRI. Proc Natl Acad Sci U S A 101, 4637-4642.

[43] Rombouts SA, Barkhof F, Goekoop R, Stam CJ, Scheltens P (2005) Altered resting state networks in mild cognitive impairment and mild Alzheimer's disease: an fMRI study. Hum Brain Mapp 26, 231-239.

[44] Filippini N, Macintosh BJ, Hough MG, Goodwin GM, Frisoni GB, Smith SM, Matthews PM, Beckmann CF, Mackay CE (2009) Distinct patterns of brain activity in young carriers of the APOE-epsilon4 allele. Proc Natl Acad Sci U S A 106, 7209-7214.

[45] Buckner RL, Snyder AZ, Shannon BJ, LaRossa G, Sachs R, Fotenos AF, Sheline YI, Klunk WE, Mathis CA, Morris JC, Mintun MA (2005) Molecular, structural, and functional characterization of Alzheimer's disease: evidence for a relationship between default activity, amyloid, and memory. $\mathrm{J} \mathrm{Neu}$ - rosci 25, 7709-7717.

[46] Miller SL, Celone K, Depeau K, Diamond E, Dickerson BC, Rentz D, Pihlajamäki M, Sperling RA (2008) Age-related memory impairment associated with loss of parietal deactivation but preserved hippocampal activation. Proc Natl Acad Sci U S A 105, 2181-2186.

[47] Lustig C, Snyder AZ, Bhakta M, O’Brien KC, McAvoy M, Raichle ME, Morris JC, Buckner RL (2003) Functional deactivations: change with age and dementia of the Alzheimer type. Proc Natl Acad Sci U S A 100, 14504-14509.

[48] Petrella JR, Prince SE, Wang L, Hellegers C, Doraiswamy PM (2007) Prognostic value of posteromedial cortex deactivation in mild cognitive impairment. PLoS ONE 2(10), e1104.

[49] Jagust W (2009) Mapping brain beta-amyloid. Curr Opin Neurol 22, 356-361. 